\title{
Symposium-in-Print
}

\section{Molecular Mechanisms of Photoaging in Human Skin In Vivo and Their Prevention by All-Trans Retinoic Acid}

\author{
Gary J. Fisher ${ }^{\star}$, Harvinder S. Talwar, Jiayuh Lin and John J. Voorhees \\ Department of Dermatology, University of Michigan, Ann Arbor, MI, USA
}

Received 9 October 1998; accepted 9 November 1998

\begin{abstract}
Solar UV radiation damages human skin, affecting skin tone and resiliency and leading to premature aging (photoaging), the symptoms of which include leathery texture, wrinkles, mottled pigmentation, laxity and sallowness. We propose that photoaging results largely from $U V$ induction of matrix metalloproteinases (MMP) that degrade skin collagen. We find that pretreatment of human skin with all-trans retinoic acid ( $R A$ ) inhibits $U V$ induction of MMP, suggesting that $t R A$ can protect against UV-induced collagen destruction and may therefore be able to lessen the effects of photoaging. The $t R A$ prevents $\mathrm{UV}$-induced accumulation of c-Jun protein, which is required for MMP gene expression. Activation of c-Jun transcriptional activity requires $\mathrm{N}$-terminal phosphorylation. The majority of c-Jun in human skin in vivo is $\mathbf{N}$ terminal phosphorylated. Topically applied $t R A$ does not inhibit $\mathbf{N}$-terminal phosphorylation by $\mathrm{UV}$-induced $\mathrm{c}$-Jun kinase activity in human skin. The $t R A$ likely acts to reduce $\mathrm{UV}$ induction of c-Jun protein by stimulating its breakdown through the ubiquitin-proteasome pathway.
\end{abstract}

\section{INTRODUCTION}

Solar UV radiation damages human skin cells, affecting skin tone and resiliency and leading to premature aging (photoaging), the symptoms of which include leathery texture, wrinkles, mottled pigmentation. laxity and sallowness (1). We further define photoaged skin by the presence of elevated matrix metalloproteinases (MMP) $\dagger$ that degrade collagen and appear long before the visible symptoms of photoaging. We have investigated the molecular mechanisms by which UV damages collagen in the dermal extracellular matrix. In

* To whom correspondence should be addressed at: Department of Dermatology, 1150 W. Medical Center Drive, Medical Science I. R6447. Ann Arbor. MI 48109-0609. USA. Fax: 734-647-0076: e-mail: dianemch (a) umich.edu

tAbbreviations: DTT. dithiothreitol: EGF, epidermal growth factor: JNK, c-Jun kinase: MAP. mitogen-activated kinase: MED, minimal erythema dose: MMP. matrix metalloproteinases: PMSF, phenylmethylsulfonyl fluoride: RAR, retinoic acid receptors; $t$ RA. all-trans retinoic acid.

1999 American Socicly for Photobiology (0031-8655/99 55.00$) \div 0.00$ contrast to the prevailing clinical approach to photoaging that focuses on treating symptoms after they have become clinically apparent, we advocate a preventive approach to treatment of photoaging of human skin before the appearance of clinical symptoms. We review evidence that topically applied all-trans retinoic acid ( $t \mathrm{RA})$, which is known to stimulate repair of photoaged skin (2), also protects against UV-induced collagen destruction and may therefore be able to lessen the effects of photoaging. Topical application of $t$ RA to skin at least $16 \mathrm{~h}$ prior to exposure effectively reduces induction of MMP.

We have proposed a working model for the pathophysiology of photoaging based on prior experiments that have studied molecular responses of human skin to a single UV exposure (3). According to this model, UV activates growth factor and cytokine receptors on keratinocytes and fibroblasts, resulting in downstream signal transduction through activation of mitogen-activated protein (MAP) kinase pathways. These signaling pathways converge in the nucleus of cells to induce c-Jun, which heterodimerizes with constitutively expressed c-Fos to form activated complexes of the transcription factor AP-1. In the dermis and epidermis, AP-1 induces expression of MMP collagenase, $92 \mathrm{kDa}$ gelatinase and stromelysin, which degrade collagen and other proteins that comprise the dermal extracellular matrix. We believe that dermal breakdown is followed by imperfect repair that yields a deficit in the structural integrity of the dermis, a solar scar. Over time, intermittent exposure to UV leads to an accumulation of solar scars that eventually manifest as wrinkled, photoaged skin. All-trans retinoic acid acts to inhibit induction of $\mathrm{c}$-Jun protein by $\mathrm{UV}$, preventing increased MMP and ensuing dermal damage.

\section{MATERIALS AND METHODS}

UV irradiation and tissue procurement. Healthy adult Caucasians with light to mild pigmentation, were irradiated with fluorescent UVB lights, as previously described (4). Wavelengths below 290 nm were removed by a Kodacel TA401/407 sheet. The UV dose that caused barely perceptible skin reddening (minimal erythema dose. MED) was determined for each subject. $24 \mathrm{~h}$ after irradiation. Irradiated and nonirradiated skin samples were obtained from each subject by keratome or punch biopsy, as described (5). The $t$ RA (Sigma Chemical Company) and its vehicle $(70 \%$ ethanol, $30 \%$ polyethyleneg]ycol, $0.05 \%$ butylated hydroxytoluenc) were applied to skin under occlusion. $48 \mathrm{~h}$ prior to UV treatment. All procedures 
involving human subjects were approved by the University of Michigan Institutional Review Board, and all subjects provided written informed consent.

Preparation of skin supernatants for biochemical analyses. Skin samples were ground by mortar and pestle under liquid nitrogen and homogenized in a Dounce tissue grinder in buffer containing $10 \mathrm{mM}$ HEPES, $1 \mathrm{~m} M$ EDTA, $5 \mathrm{~m} M$ EGTA, $10 \mathrm{~m} M \mathrm{MgCl}_{2}$, $50 \mathrm{~m} M$ glycerophosphate, $5 \mathrm{~m} M \mathrm{NaVO}_{4}, 2 \mathrm{~m} M$ dithiothreitol (DTT), $0.5 \mathrm{~m} M$ phenylmethylsulfonyl fluoride (PMSF), $10 \mu \mathrm{g} / \mathrm{mL}$ aprotinin, $10 \mu \mathrm{g} /$ $\mathrm{mL}$ leupeptin, $10 \mu \mathrm{g} / \mathrm{mL}$ pepstatin and $0.5 \%$ Nonidet $\mathrm{P}-40$. Homogenates were centrifuged at $14000 \mathrm{~g}$ for $15 \mathrm{~min}$, supernatants collected and used for biochemical determinations described below.

$c$-Jun kinase ( JNK) assay. The JNK activity in skin supernatants was determined by solid-phase kinase assay, as described (6); JNK was immunoprecipitated with antibodies from Santa Cruz Biotechnology and assayed for activity using glutathione S-transferase-cJun as substrate.

Preparation of nuclear extracts from human skin. Individual keratome biopsies were placed in $0.25 \%$ trypsin, $0.1 \%$ EDTA for 40 min at $37^{\circ} \mathrm{C}$. Skin samples were trypsinized and cells were released from the tissue by scraping. Released cells were washed three times in phosphate-buffered saline, and resuspended in $20 \mathrm{mM}$ Tris $(\mathrm{pH}$ 8.0), $20 \mathrm{mM} \mathrm{NaCl}, 6 \mathrm{mM} \mathrm{MgCl}, 0.2 \%$ Triton X-100. $1 \mathrm{~m} M$ DTT, $200 \mathrm{~m} M$ sucrose, $1 \mathrm{mM}$ PMSF, $0.02 \mathrm{mg} / \mathrm{mL}$ leupeptin, $0.02 \mathrm{mg} / \mathrm{mL}$ pepstatin A, at a concentration of $2 \times 10^{7}$ cells $/ \mathrm{mL}$ (7). Nuclear extracts containing $\mathrm{c}$-Jun were prepared as described previously (8). Extracts were aliquoted and stored at $-70^{\circ} \mathrm{C}$ prior to use.

Western analysis of $c_{-}-J u n$. The c-Jun protein (antibody from Transduction Laboratories) and phosphorylated c-Jun (antibody from New England BioLabs) were detected in nuclear extracts from human skin by western analysis, as described (7).

\section{RESULTS AND DISCUSSION}

\section{UV triggers MAP kinase signal transduction pathways in human skin in vivo}

Cellular physiology is largely regulated by hormones, growth factors and cytokines acting through their respective cell surface receptors. One of the earliest effects of UV is activation of cell surface receptors (9). It appears likely that UV activates most, if not all, growth factor and cytokine receptors. We examined activation of the epidermal growth factor (EGF) receptor by UV in human skin. Activation of the EGF receptor, as demonstrated by autophosphorylation of specific tyrosine residues, occurs within $15 \mathrm{~min}$ of UV irradiation (2 MED) and remains elevated for at least $2 \mathrm{~h}$ (10).

Activation of growth factor and cytokine receptors results in assembly of protein complexes that transduce signals from the cell surface to the nucleus. When these complexes assemble, small, GTP-binding regulatory proteins are activated, including Ras and Rac, that function as molecular switches to trigger MAP kinase signal transduction pathways. In human skin, Ras is activated within $30 \mathrm{~min}$ of UV exposure and remains activated for at least $24 \mathrm{~h}$ (10). Activated Rac directly stimulates the NADPH oxidase enzyme complex in cells, increasing levels of two reactive oxygen species, superoxide anion and hydrogen peroxide that are critical for MAP kinase-mediated signal transduction.

Ultraviolet irradiation activates each of the three MAP kinases, ERK, JNK and p38, through dual phosphorylation of threonine and tyrosine. The ERK, JNK and p38 are activated within $1 \mathrm{~h}$ of $\mathrm{UV}$ irradiation, maximally activated 4 h post-UV and return to baseline within $24 \mathrm{~h}$ after UV exposure (10).
UV exposure induces c-Jun and activates AP-1 in human skin in vivo

Activation of ERK, JNK and p38 MAP kinases results in increased expression of the transcription factors c-Fos and c-Jun in cultured cells. The c-Fos and c-Jun form stable heterodimers, and, together with other protein factors, form the transcription factor AP-1. The AP- 1 regulates the genes for several members of the MMP family (11). In nonirradiated human skin, c-Jun mRNA and protein levels are minimally detectable, while c-Fos mRNA and protein are readily detectable. Ultraviolet rapidly induces c-Jun mRNA and protein, but c-Fos protein is not affected (10). Our findings indicate that AP-1 activation by UV is primarily dependent on induction of c-Jun, because c-Fos is constitutively expressed in human skin in vivo.

This conclusion is further supported by measurement of AP-1 complexes and by gel shift analyses in nonirradiated and UV-irradiated human skin. In nonirradiated human skin, AP-1 complexes contain predominantly c-Fos and Jun D, which, like c-Fos, is constitutively expressed and not induced by UV irradiation (10). Following UV irradiation, AP1 levels are increased and AP-1 complexes are enriched in $c-J u n$, relative to nonirradiated human skin. These findings indicate that UV-induced c-Jun forms functional complexes with constitutively expressed c-Fos (10). The UV activation of AP-1, measured by gel shift analyses, is induced at very low levels of UV (0.1-1 MED). Taken together, these results indicate that UV induces increases in activated AP-1 complexes, composed of c-Jun and c-Fos, in human skin in vivo. Activated AP-1 would be expected to stimulate transcription of MMP target genes.

\section{UV irradiation induces MMP that degrade the dermal extracellular matrix in human skin in vivo}

The MMP are a family of zinc-requiring enzymes that specifically break down proteins that comprise connective tissue. The AP-1 strongly regulates transcription of at least four members of the MMP family, collagenase (that cleaves fibrillar types I and III collagens), $92 \mathrm{kDa}$ gelatinase (that further breaks down collagen subsequent to collagenase cleavage, and also degrades type IV collagen and elastin), stromelysin (that degrades type IV collagen, elastin and other extracellular matrix molecules, including proteoglycans and laminins) and metalloelastase (that cleaves elastin fibers) (11). Ultraviolet induces expression of these MMP genes in human skin in vivo, consistent with UV activation of AP-1. Collagenase, $92 \mathrm{kDa}$ gelatinase and stromelysin mRNA levels are increased following UV irradiation and remain elevated for more than $24 \mathrm{~h}$ (4). These data are consistent with the notion that UV activation of AP-1 drives MMP induction in human skin in vivo.

Sun damage to the extracellular matrix of the dermis becomes histologically and clinically observable only after it accumulates with multiple sun exposures (1). A single UV exposure causes acute induction of MMP, which return to basal levels within $48-72 \mathrm{~h}$ after exposure. If MMP induction by UV is responsible for dermal damage in photoaging, then MMP induction would be expected to remain responsive to multiple UV exposures. To test this hypothesis, subjects were irradiated multiple times at $48 \mathrm{~h}$ intervals over a 
7 day period. As expected, collagenase and $92 \mathrm{kDa}$ gelatinase were maximally induced following a single exposure to UV. With every-other-day exposure to UV, these maximal levels were maintained for 7 days, suggesting that there is no attenuation of MMP induction with repeated exposures (3).

In order for UV-induced MMP to cause the dermal damage known as photoaging, they must be localized in the dermis. We utilized in situ hybridization, immunohistology and in situ zymography to localize expression and activity of MMP in nonirradiated and UV-irradiated human skin. While mRNA, proteins and activities of collagenase, $92 \mathrm{kDa}$ gelatinase and stromelysin were minimally detectable in nonirradiated human skin with these techniques, mRNA and activities of these MMP were readily detectable following exposure to UV. Collagenase, $92 \mathrm{kDa}$ gelatinase and stromelysin mRNA were induced throughout all of the layers of the epidermis, but only collagenase and stromelysin mRNA were induced in dermal cells. Activities of these MMP were also found in the dermis. Together, these data indicated that collagenase, $92 \mathrm{kDa}$ gelatinase and stromelysin proteins are substantially elevated and active in the dermis and epidermis of human skin following UV irradiation (3).

Together, collagenase, $92 \mathrm{kDa}$ gelatinase and stromelysin have the capacity to degrade all of the protein components of the dermal extracellular matrix. To obtain direct evidence for collagen breakdown following acute UV exposure, we quantitated soluble breakdown products of type I fibrillar collagen in nonirradiated and UV-irradiated human skin by radioimmunoassay. Results indicated that the MMP induced by UV irradiation and localized in the dermis degrade endogenous fibrillar collagen in human skin in vivo (3).

In addition to inducing AP-1-regulated MMP, UV irradiation induces an endogenous MMP inhibitor, TIMP-1 (3). The TIMP-I mRNA and protein are induced with similar kinetics and UV dose dependence as the MMP. This coinduction of MMP and TIMP- 1 acts to inhibit MMP activity and thereby prevent excessive connective tissue break down (12). Data presented above, however, indicate that UV-induced MMP activities exceed the inhibitory capacity of TIMP-1, and therefore some dermal extracellular matrix destruction occurs

\section{tRA pretreatment inhibits UV induction of MMP by inhibiting $U V$ induction of $c$-Jun protein in human skin in vivo}

Because UV induces AP-1, which stimulates expression of MMP that degrade collagen in the dermis of human skin in vivo, preventive agents must block induction at some point along the signal transduction pathway. In cultured cells, $t \mathrm{RA}$ antagonizes AP-1 through a mechanism known as "transrepression" that is mediated by nuclear retinoic acid receptors (RAR) (13). Evidence suggests that transrepression of AP- 1 by $t$ RA-activated RAR results from binding of protein factors required for AP- 1 activity to activated RAR. We therefore examined the effects of $t R A$ on $U V$ induction of MMP in human skin in vivo. Pretreatment of skin with $t \mathrm{RA}$ significantly inhibited UV induction of collagenase, $92 \mathrm{kDa}$ gelatinase and stromelysin mRNA $(50-60 \%)$ and proteins $(60-80 \%)(4)$.

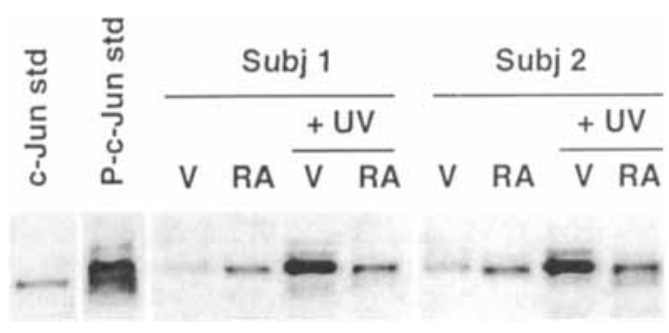

Figure 1. The c-Jun in human skin in vivo, whether exposed to UV or $t \mathbf{R A}$, exists in a predominantly $\mathrm{N}$-terminal phosphorylated state Human buttock skin was pretreated with vehicle (V) or $0.1 \% t$ RA (RA) for $48 \mathrm{~h}$ prior to irradiation with 2 MED UVB (UV). Biopsies were taken $8 \mathrm{~h}$ later and nuclear extracts were prepared and analyzed for c-Jun protein levels by western blot analysis using an antibody against total c-Jun. Lanes 1 and 2 show recombinant nonphosphorylated and N-terminal phosphorylated c-Jun standards, respectively. Western blots of nuclear extracts prepared from two different individuals that are representative of six different subjects are shown.

The $t$ RA inhibited UV-induced MMP activities throughout the epidermis and dermis, as demonstrated both by immunohistology and in situ zymography (3). Interestingly, tRA did not inhibit UV induction of TIMP-1 (3). Therefore, $t$ RA pretreatment increased the ratio of TIMP-1 to MMP in human skin following UV irradiation. Elevated TIMP-1 would effectively inhibit most, if not all, of the residual MMP activities induced by UV in $t$ RA-pretreated skin. This relative TIMP-1 excess may explain why $t \mathrm{RA}$ pretreatment appears to inhibit UV induction of MMP activities completely, as measured by in situ zymography. These data raise the possibility that $t \mathrm{RA}$, which has previously been shown to improve the appearance of photodamaged skin, may also prevent photodamage.

Because UV induction of AP-1 appears to be critical for increased MMP expression, we examined the effect of $t R A$ on AP- 1 levels. Pretreatment of human skin with $t$ RA for 48 $h$ resulted in $70 \%$ inhibition of UV-induced AP-1 DNA binding. In marked contrast, $8 \mathrm{~h}$ of $t \mathrm{RA}$ pretreatment had no effect on UV-induced AP-1 DNA binding (10).

We next examined the effect of $t$ RA pretreatment on levels of c-Fos and c-Jun in nonirradiated and UV-irradiated human skin. Pretreatment with $t$ RA for $48 \mathrm{~h}$ did not alter the levels of c-Fos mRNA or c-Jun mRNA or protein in nonirradiated human skin, or c-Fos mRNA or protein levels in UV-irradiated skin. As stated earlier, UV induces c-Jun mRNA and protein. Pretreatment with $t$ RA for $48 \mathrm{~h}$ did not inhibit UV induction of c-Jun mRNA but did inhibit UV induction of c-Jun protein by $70 \%$ throughout the epidermis and dermis. Eight hours of $t$ RA pretreatment did not inhibit UV induction of c-Jun protein (10). Taken together, these data suggest a mechanism whereby $t \mathrm{RA}$, acting through its nuclear receptors, induces expression of an inhibitor of $c$ Jun protein translation or an activator of c-Jun degradation.

Recent evidence indicates that the ubiquitin proteasome pathway represents a major route for c-Jun degradation. The $\mathrm{N}$-terminal phosphorylation of c-Jun by JNK inhibits c-Jun ubiquitination and thereby prolongs the half-life of c-Jun (14). In nontreated and UV-irradiated human skin, the majority of $\mathrm{c}$-Jun is N-terminal phosphorylated (Fig. 1). The majority of c-Jun in human skin treated with tRA alone or tRA plus UV irradiation is also $\mathrm{N}$-terminal phosphorylated (Fig. 1) Thus, whether c-Jun is present in low basal levels 


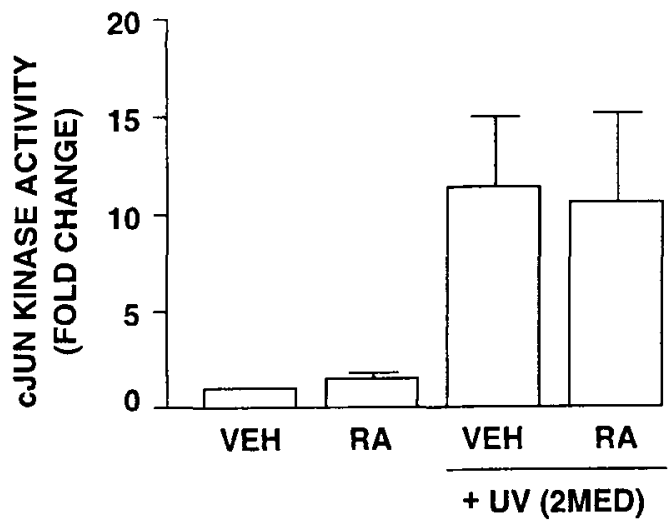

Figure 2. The $t$ RA does not inhibit UV-induced JNK activity in human skin in vivo. Human buttock skin was pretreated with vehicle (veh) or $0.1 \% t$ RA (RA) for $24 \mathrm{~h}$, then irradiated with 2 MED UVB (UV) and biopsies taken $4 \mathrm{~h}$ later. The JNK activity was measured in the supernatants prepared from the biopsies, using glutathionine $\mathbf{S}$-transferase-c-Jun as the substrate. Data are expressed as fold change in activity compared to vehicle-treated skin $(n=7)$.

or high UV-induced levels, it is predominantly N-terminal phosphorylated in human skin in vivo. This is consistent with $\mathrm{N}$-terminal phosphorylation stabilizing $\mathrm{c}$-Jun. As indicated above, UV induces JNK-catalyzed c-Jun phosphorylation and elevates c-Jun protein levels for at least $24 \mathrm{~h}$. It has been reported that $t$ RA inhibits JNK in cultured cells $(15,16)$. We found, however, that $t$ RA did not inhibit UV-induced JNK activity in human skin (Fig. 2). We speculate that $t$ RA acts through stimulation of c-Jun dephosphorylation and/or ubiquitination. In either case, nonphosphorylated c-Jun would be ubiquitinated and degraded and therefore could not accumulate. We are investigating these possibilities.

\section{CONCLUDING REMARKS}

The above findings present a framework for understanding how solar UV radiation damages collagen in human skin. Ultraviolet appears to act like a universal ligand to activate cell surface receptors that, once activated, trigger downstream signaling pathways that in turn regulate the activity of different transcription factors, thereby altering expression of many genes, including AP-1-regulated members of the MMP family. The UV-induced MMP are induced throughout the epidermis and dermis, where they degrade collagen and the other proteins of the dermal extracellular matrix. Matrix degradation is succeeded by imperfect repair, termed solar scarring, a process that occurs with each exposure to UV. Because solar scar formation is likely to occur even at low doses of UV that do not cause skin reddening, even minimal sun exposure will lead to photoaging over many years' time.

The results of these studies have also provided a theoretical foundation for developing new clinical preventive agents that could block UV-induced responses at the level of cell surface receptors, protein kinase cascades, transcription factors or MMP. Understanding the pathophysiology of photoaging and the pharmacological agents that can prevent it may also provide insight into other human maladies, because inappropriate activation of cell surface receptors, signal transduction pathways, transcription factors and MMP occur in a variety of pathological conditions characterized by hyperproliferation, malignant transformation, inflammation and connective tissue damage.

\section{REFERENCES}

1. Gilchrest, B. A. and M. Yaar (1992) Aging and photoaging of the skin: observations at the cellular and molecular level. $B r . J$. Dermatol. 127, 25-35.

2. Ellis, C. N., J. S. Weiss, T. A. Hamilton, J. T. Headington, A S. Zelickson and J. J. Voorhees (1990) Sustained improvement with prolonged topical tretinoin (retinoic acid) for photoaged skin. J. Am. Acad. Dermatol. 23, 629-637.

3. Fisher, G. J., Z. Q. Wang, S. C. Datta, J. Varani, S. Kang and J. J. Voorhees (1997) Pathophysiology of premature skin aging induced by ultraviolet light. $N$. Engl. J. Med. 337, 1419-1428.

4. Fisher, G. J., S. C. Datta, H. S. Talwar, Z. Q. Wang, J. Varani, S. Kang and J. J. Voorhees (1996) Molecular basis of sun-induced premature skin ageing and retinoid antagonism. Nature 379, 335-379.

5. Fisher, G, J., J. Esmann, C. E. Griffiths, H. S. Talwar, E. A Duell, C. Hammerberg, J. T. Elder, L. J. Finkel, G. D. Karabin and B. J. Nickoloff (1991) Cellular, immunologic and biochemical characterization of topical retinoic acid-treated human skin. J. Invest. Dermatol. 96. 699-707.

6. Hibi, M., A. Lin, T. Smeal, A. Minden and M. Karin (1993) Identification of an oncoprotein- and UV-responsive protein $\mathrm{ki}$ nase that binds and potentiates the c-Jun activation domain. Genes \& Dev. 7. 2135-2148.

7. Fisher, G. J., H. S. Talwar, J. H. Xiao, S. C. Datta, A. P. Reddy, M. P. Gaub, C. Rochette-Egly, P. Chambon and J. J. Voorhees (1994) Immunological identification and functional quantitation of retinoic acid and retinoid $X$ receptor proteins in human skin. J. Biol. Chen. 269, 20629-20635.

8. Dignam, J. D., R. M. Lebovitz and R. G. Roeder (1983) Accurate transcription initiation by RNA polymerase II in a soluble extract from isolated mammalian nuclei. Nucleic Acids Res. 11, $1475-1489$.

9. Rosette, C. and M. Karin (1996) Ultraviolet light and osmotic stress: activation of the JNK cascade through multiple growth factor and cytokine receptors. Science 274, I194- I 197.

10. Fisher, G. J., H. S. Talwar, J. Lin, P. Lin, F. McPhillips, Z. Q. Wang, X. Li, Y. Wan, S. Kang and J. J. Voorhees (1998) Retinoic acid inhibits induction of $\mathrm{c}$-Jun protein by uitraviolet radiation that occurs subsequent to activation of mitogen-activated protein kinase pathways in human skin in vivo. J. Clin. Invest. 101, 1432-1440.

I I. Angel, P. and M. Karin (1992) Specific members of the Jun protein family regulate collagenase expression in response to various extracellular stimuli. Matrix Suppl. 1. 156-164.

12. Borden, P. and R. A. Heller (1997) Transcriptional control of matrix metalloproteinases and tissue inhibitors of matrix metalloproteinases. Crit. Rev. Eukaryotic Gene Expression 7, 159178.

13. Chen, J. Y.. S. Penco, J, Ostrowski, P. Balaguer, M. Pons, J. E. Starrett, P. Reczek, P. Chambon and H. Gronemeyer (1995) RAR-specific agonists/antagonists which dissociate transactivation and AP-1 transrepression, inhibit anchorage-independent cell proliferation. EMBO J. 14, 1187-1197.

14. Musti, A. M., M. Treier and D. Bohmann (1997) Reduced ubiquitin-dependent degradation of c-Jun after phosphorylation by MAP kinases. Science 275, 400-402.

15. Lee, H. Y., G. L. Walsh, M. I. Dawson, W. K. Hong and J. M. Kurie (1998) All-trans retinoic acid inhibits Jun N-terminal kinase-dependent signaling pathways. J. Biol. Chem. 273, 70667071.

16. Caelles, C., J. M. Gonzalez-Sancho and A. Munoz (1997) Nuclear hormone receptor antagonism with AP-1 by inhibition of the JNK pathway. Genes \& Dev. 11, 3351-3364. 